\title{
TROMBOSIS DE VENA CAVA INFERIOR EN EMBARAZO A TÉRMINO: REPORTE DE CASO Y REVISIÓN DE LA LITERATURA
}

\section{Inferior vena cava thrombosis in term pregnancy: Case report and review of the literatura}

María Fernanda Escobar-Vidarte, MD, MSC'; Sara del Pilar Loaiza-Osorio, MD $^{2}$; Albaro José Nieto-Calvache, $M D^{1}$

Recibido: mayo 12/17 - Aceptado: agosto 29/18

\section{RESUMEN}

Objetivo: realizar una revisión de la literatura publicada sobre el tratamiento de la trombosis de vena cava inferior en las gestantes.

Materiales y métodos: se describe un caso de trombosis de vena cava inferior en embarazo a término que consultó a una institución privada de cuarto nivel de complejidad, centro de referencia. Se manejó inicialmente con heparina de bajo peso molecular, suspendida 24 horas antes del parto. Se obtuvo un buen resultado perinatal, aunque presentó hemorragia posparto y hematoma perineal. Posteriormente se hizo manejo endovascular para trombolisis e intento de trombectomía. Se realizó una revisión de la literatura con los términos "vena cava inferior", "filtros de vena cava", "trombosis" y "embarazo". Se incluyeron reportes de caso y series de casos en inglés y español. Se seleccionaron todos los casos que describieran el tratamiento utilizado en mujeres con trombosis de vena cava inferior, diagnosticadas durante el embarazo o el puerperio. Resultados: se incluyeron 17 publicaciones que

1 Unidad de alta complejidad obstétrica, Departamento de Ginecología y obstetricia, Fundación Valle del Lili, Cali (Colombia).

2 Centro de investigaciones clínicas, Fundación Valle del Lili, Cali (Colombia)._saraloaiza1@gmail.com cumplieron con los criterios de búsqueda. Se identificaron 41 casos; 35 pacientes fueron diagnosticadas con trombosis de vena cava inferior durante el embarazo y 6 durante el puerperio. El $100 \%$ de las pacientes fueron anticoaguladas con heparina de bajo peso molecular o heparina no fraccionada, 34 gestantes requirieron filtro de vena cava inferior para manejo periparto y solo una gestante terminó en trombectomía.

Conclusión: la trombosis de vena cava inferior en el periparto es un reto desde el punto de vista terapéutico, se debe equilibrar el riesgo de TEP y hemorragia masiva. Es fundamental tener una adecuada coordinación entre los diferentes servicios implicados garantizando máxima seguridad para evitar complicaciones.

Palabras clave: terapéutica, trombosis de la vena, vena cava inferior, embarazo.

\section{ABSTRACT}

Objective: To conduct a review of the published literature on the treatment of inferior vena cava thrombosis in pregnancy.

Materials and methods: Case description of inferior vena cava thrombosis in a woman with term pregnancy coming to a private level IV referral 
centre. It was initially managed with low-molecular weight heparin, which was discontinued 24 hours before delivery. Although the perinatal outcome was good, the patient developed postpartum bleeding and perineal haematoma. Endovascular treatment for thrombolysis and attempted thrombectomy was performed later. A review of the literature was conducted using the terms "inferior vena cava", "vena cava filters", "thrombosis" and "pregnancy". Case reports and case series in English and Spanish were included. All the cases describing the treatment used in women with vena cava thrombosis diagnosed during pregnancy or the postpartum period were selected.

Results: Overall, 17 publications that met the search criteria were included, and 41 cases were identified: 35 patients were diagnosed with inferior vena cava thrombosis during pregnancy and 6 were identified during the postpartum period. Low molecular weight heparin or unfractionated heparin was used for anticoagulation in $100 \%$ of the patients; 34 pregnant women required inferior vena cava filter for peripartum management, and only one patient needed thrombectomy.

Conclusion: Peripartum inferior vena cava thrombosis is challenging from the therapeutic point of view. There is a need to balance the risk of pulmonary thromboembolism and massive bleeding. Adequate coordination between the different services involved is mandatory in order to ensure maximum safety and avoid complications.

Key words: Therapeutics, vein thrombosis, inferior vena cava, pregnancy.

\section{INTRODUCCIÓN}

La enfermedad tromboembólica venosa (ETV) es una entidad que incluye la trombosis venosa profunda (TVP) y el tromboembolismo pulmonar (TEP). La TVP es definida como la formación de un trombo en el interior de los vasos venosos, con reacción inflamatoria en la pared vascular; el TEP se define como la oclusión total o parcial de la circulación arterial pulmonar por un coágulo sanguíneo proveniente de la circulación venosa sistémica (1, 2). En algunos países se ha descrito que la ETV constituye la principal causa de morbi-mortalidad materna, siendo la causa de complicación de 1-2 por cada 1000 embarazos $(3,4)$ Heit et al. informan que la incidencia global de ETV asociada al embarazo es de aproximadamente 200 por 100.000 años-mujer (5). Ellos también informan que el riesgo de ETV en el embazo es de 4,3 en relación con las mujeres no embrazadas, similar al riesgo de TVP de 4,6 descrito por Pomp et al. (6), ambos en relación con las mujeres no embarazadas. Este último autor describe que el riesgo de ETV en los tres meses siguientes al parto puede incrementarse hasta 60 veces. Por otra parte, Jacobsen et al. describen que cerca del $50 \%$ de los casos de ETV se presentan en las seis semanas siguientes al parto (7).

El embarazo está asociado a un estado de hipercoagulabilidad, el cual es multifactorial y se piensa que sea debido a la combinación de factores hormonales y físicos, como también a cambios hematológicos, los cuales son fundamentales en la patogénesis de la trombosis intravascular, estos incluyen: estasis venosa, daño de la pared vascular y cambios en la coagulabilidad de la sangre (8). Entre los factores de riesgo asociados a la ETV en el embarazo se encuentran los de origen hereditario, tales como: deficiencia de antitrombina III, deficiencia de proteína $\mathrm{C}$, deficiencia de proteína $\mathrm{S}$, resistencia a la proteína $\mathrm{C}$ activada, mutación de la protrombina 20210 e hiperhomocisteinemia (9). De igual forma, existen factores de riesgo adquiridos que pueden concomitar durante el embarazo, por ejemplo: edad, presencia de anticuerpos antifosfolípidos, historia de tromboflebitis superficial y TEV, inmovilidad, obesidad y viajes prolongados (10).

El diagnóstico del tromboembolismo venoso durante el embarazo es un reto, porque los síntomas clásicos son menos específicos durante este estado que en la mujer no embarazada. Los síntomas y signos normales durante la gestación que pueden simular una ETV incluyen: dolor y edema o hinchazón de la pierna, disnea, taquipnea, taquicardia, 
palpitaciones (1). Durante el embarazo, la trombosis comienza con más frecuencia en las venas de la pantorrilla y el sistema venoso profundo ilio-femoral, con una fuerte predilección por el miembro inferior izquierdo. La afección de esta extremidad se debe al parecer a la compresión que ejerce la arteria iliaca derecha sobre la vena iliaca izquierda en el sitio donde se cruzan; además, cuando una mujer en estado grávido permanece en posición supina, la velocidad de flujo sanguíneo disminuye, en particular en la pierna izquierda, lo que posiblemente contribuye a mayor incidencia de trombosis venosa profunda en esta extremidad (11).

En cuanto al tratamiento médico y quirúrgico de la ETV y TEP en el embarazo hay mucha controversia. Por ejemplo, en el manejo inicial se ha sugerido el uso de heparina no fraccionada (HNF) ya que permite la reversión rápida de la anticoagulación debido a que la heparina se elimina de manera eficiente por su unión al endotelio (8). Con la HNF pueden presentarse riesgos debido a que, como su metabolismo ocurre principalmente a través del hígado y el riñón, en caso de falla de cualquiera de estos dos órganos los niveles de heparina plasmática se encontrarían anormalmente elevados, además es un inhibidor competitivo de la adenosintrifosfatasa que hace más lenta la reparación del endotelio celular (12).

Otros autores recomiendan utilizar las heparinas de bajo peso molecular por tener una vida media de 4 a 5 horas, no estar asociadas a efectos adversos fetales, tener mayor actividad contra el factor Xa, y unirse menos a proteínas plasmáticas, células endoteliales y macrófagos, con lo que aumentan su biodisponibilidad, vida media y actividad anticoagulante (13). Sin embargo, la dosificación de la heparina de bajo peso molecular se debe ajustar de acuerdo al peso de cada paciente o a los niveles de antifactor Xa (14). Por otra parte, no hay claridad sobre el momento de suspender la heparina antes del parto. Por ejemplo, Ginsberg et al. recomiendan la suspensión de la heparina no fraccionada intravenosa de 4 a 6 horas antes de la hora prevista para la cirugía y control del tiempo de tromboplastina parcial activado (TTPA) antes de la incisión (15). Respecto al uso de agentes trombolíticos hay dudas sobre su indicación en la gestante debido a que esta terapia ha sido asociada a riesgos de sangrado, teratogénesis y pérdida fetal, y está reservada para casos de TEP con choque o paro cardiaco $(16,17)$. Por último respecto al uso de los filtros colocados en la vena cava inferior es poca la experiencia respecto a su uso en la gestante $(18,19)$.

El objetivo de la presentación del caso de una gestante con TVP de la vena cava en el último trimestre del embarazo es realizar una revisión de la literatura publicada sobre el tratamiento de esta patología en gestantes.

\section{PRESENTACIÓN DEL CASO}

Lugar: Fundación Valle del Lili, institución de cuarto nivel de complejidad, centro de referencia médica en el suroccidente colombiano, ubicada en la ciudad de Cali (Colombia), que atiende población del régimen de aseguramiento contributivo y privado en el Sistema General de Seguridad Social en Salud (SGSS) de Colombia.

Mujer de 33 años de edad, gesta 2, partos 1, vivos 1 , que consultó a esta institución hospitalaria a las 37,2 semanas por cuadro clínico de 3 días de evolución consistente en dolor intenso en región inguinal izquierda asociado a actividad uterina irregular. Al examen físico de ingreso presentó: frecuencia respiratoria, 18 respiraciones por minuto (rpm); frecuencia cardiaca, 87 latidos por minuto (lpm); tensión arterial (TA) de 132/71 mm/Hg, y saturación de oxígeno del $92 \%$. Al evaluar las extremidades se observó edema marcado en la extremidad inferior izquierda, con asimetría en diámetro de ambas piernas de $3 \mathrm{~cm}$, siendo de mayor tamaño la pierna izquierda. La gestante refirió como antecedentes de importancia un bypass gástrico que se asoció a pérdida de 50 kilos en 5 años y retardo de crecimiento intrauterino (RCIU) en embarazo previo, que fue atendido por cesárea. Durante su nueva gestación, la paciente fue diagnosticada con diabetes gestacional y anemia ferropénica moderada. 
Por alta sospecha clínica de TVP se realizó ecografía doppler de miembros inferiores que reportó TVP activa de unión safeno-femoral y femoral común izquierda, por lo cual se inició heparina de bajo peso molecular (HBPM) a dosis de $1 \mathrm{mg} / \mathrm{kg}$ cada $12 \mathrm{~h}$. Posteriormente, la paciente presentó regularización de actividad uterina; ante el riesgo de sangrado masivo por la anticoagulación y la posibilidad de TEP masivo por descompresión uterina, se consideró colocación de filtro de vena cava temporal, sin embargo, durante el procedimiento se evidenció que la trombosis se extendía hasta la vena cava inferior (figura 1), contraindicándose su implantación.

Ante la urgencia del manejo definitivo con anticoagulación y trombolisis local se decidió suspender la heparina de bajo peso molecular 24 horas antes de que la paciente iniciara fase activa del trabajo de parto. Se indujo parto vaginal con balón de dilatación endocervical y conducción con oxitocina, y se inició dexmetomidina como premedicación ante posible uso de trombolisis. La paciente pudo deambular, se le permitió la vía oral y recibió manejo médico para el dolor en miembro inferior con hidromorfona vía oral y acetaminofén endovenoso. Al segundo día de hospitalización se atendió parto instrumentado, con espátulas de Velasco, para acortar el periodo de expulsivo. Se obtuvo recién nacido de sexo masculino de $2900 \mathrm{~g}$ de peso y APGAR 10-10.

Inmediatamente después del nacimiento la paciente presentó hemorragia posparto (HPP) secundaria a hipotonía uterina. Los signos vitales evidenciaron frecuencia cardiaca de $101 \mathrm{lpm}$, frecuencia respiratoria de $23 \mathrm{rpm}$, tensión arterial de 108/49 mm/hg con tensión arterial media de 68 y saturación del 99\%; el sangrado visual estimado fue de 1000 cc. Se activó código rojo obstétrico con uso de 40 unidades endovenosas de oxitocina, 0,2 mg de methergin intramuscular y $800 \mathrm{mcg}$ intrarrectales de misoprostol (dosis acordes a protocolo institucional), colocación de balón de Bakri y traje antichoque.

A los 90 min posparto la paciente se encontraba hemodinámicamente estable y sin sangrado activo; sin embargo, los signos vitales evidenciaron taquicardia asociada a dolor torácico, tos y desaturación (<90 SO2), por lo cual se sospechó TEP y se inició anticoagulación con heparina no fraccionada (2400

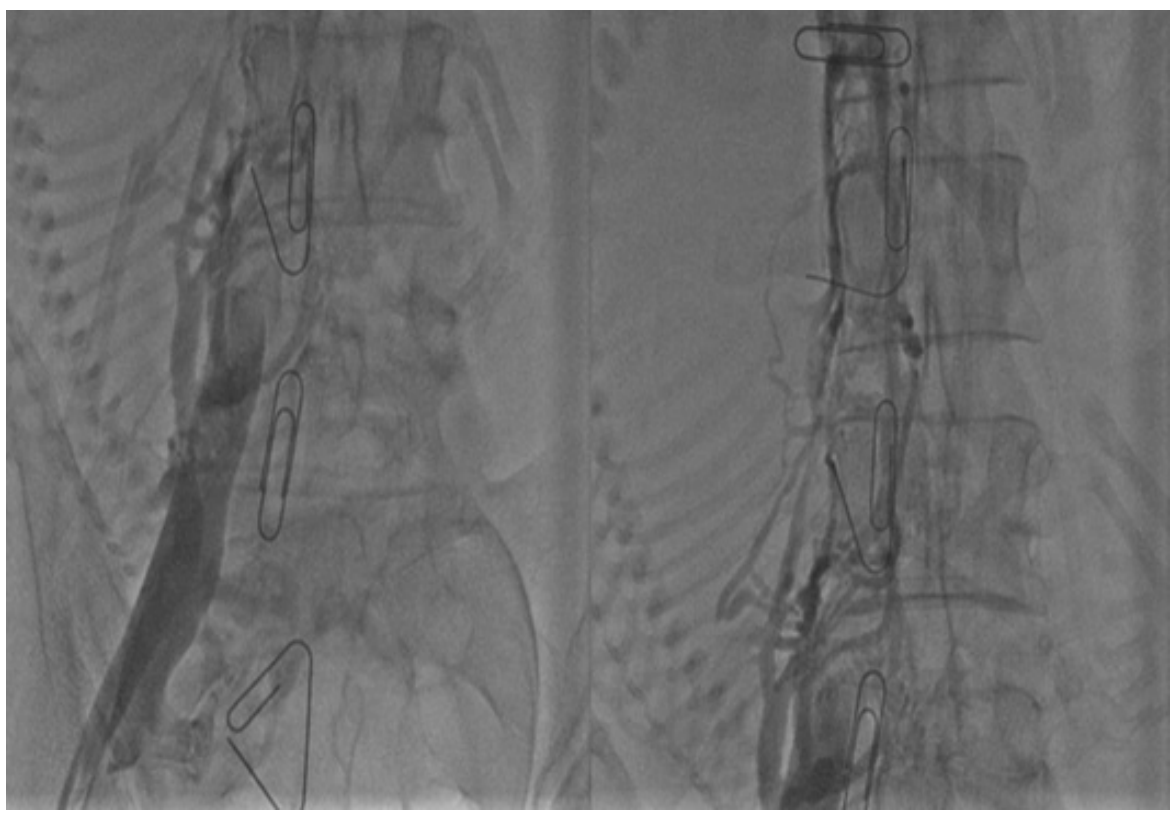

Figura 1 A. Flebografía de vena iliaca externa y vena cava inferior. B. Defecto de llenado por trombosis de vena cava inferior (imágenes autorizadas por servicio de hemodinamia, Fundación Valle del Lili). 
unidades por hora); el servicio de hematología prefirió dicha anticoagulación debido a que su efecto podía ser revertido, con una actividad máxima a las dos horas de su administración; el angio TAC pulmonar realizado a las 3 horas del evento descartó TEP no masivo.

Durante la evolución clínica (96 horas después del parto) presentó un hematoma a nivel de la episiotomía, con extensión perineal; requirió drenaje quirúrgico y empaquetamiento vaginal, con resolución en las siguientes 48 h; por difícil y lenta anticoagulación con heparina no fraccionada (HU), debido a que presentó niveles insuficientes en el tiempo parcial de tromboplastina (PTT), se consideró cambiar a heparina de bajo peso molecular (enoxaparina a $1 \mathrm{mg} / \mathrm{kg}$ cada $12 \mathrm{~h}$ ).

El día 11 del puerperio se decide realizar trombolisis local, la flebografía corrobora trombosis de todo el eje ilio cava izquierdo. Previo consentimiento informado, se decidió recanalizar todo el eje venoso hasta la cava retrohepática y se realizó trombolisis con alteplasa en bolo inicial de $4 \mathrm{mg}$, con goteo de mantenimiento a razón de $1,5 \mathrm{mg} / \mathrm{h}$. Durante el procedimiento no fue suspendida la heparina de bajo peso molecular. A las $30 \mathrm{~h}$ de la trombolisis presentó sangrado vaginal y frecuencia cardiaca de $91 \mathrm{lpm}$, tensión arterial de 80/40 mm/ hg, frecuencia respiratoria de $22 \mathrm{rpm}$ y saturación de oxígeno del $92 \%$. Se diagnosticó choque hipovolémico por hematoma disecante del canal vaginal; se decidió interconsulta con el servicio de hematología, quienes ordenaron realizar transfusión de 2 unidades de glóbulos rojos por caída de hemoglobina mayor a $2 \mathrm{~g}$, reanimación con líquidos cristaloides, empaquetamiento vaginal y colocación de balón de Backri. A las doce horas posteriores, por orden del servicio de hematología, se decide iniciar manejo con enoxaparina a $1 \mathrm{mg} / \mathrm{kg}$ cada $12 \mathrm{~h}$, sin nuevos episodios de sangrado. Se eligió la heparina de bajo peso molecular debido a que la incidencia de eventos tromboembólicos venosos recurrentes es menor en personas tratadas con enoxaparina que con heparina no fraccionada. Se llevó a flebografía

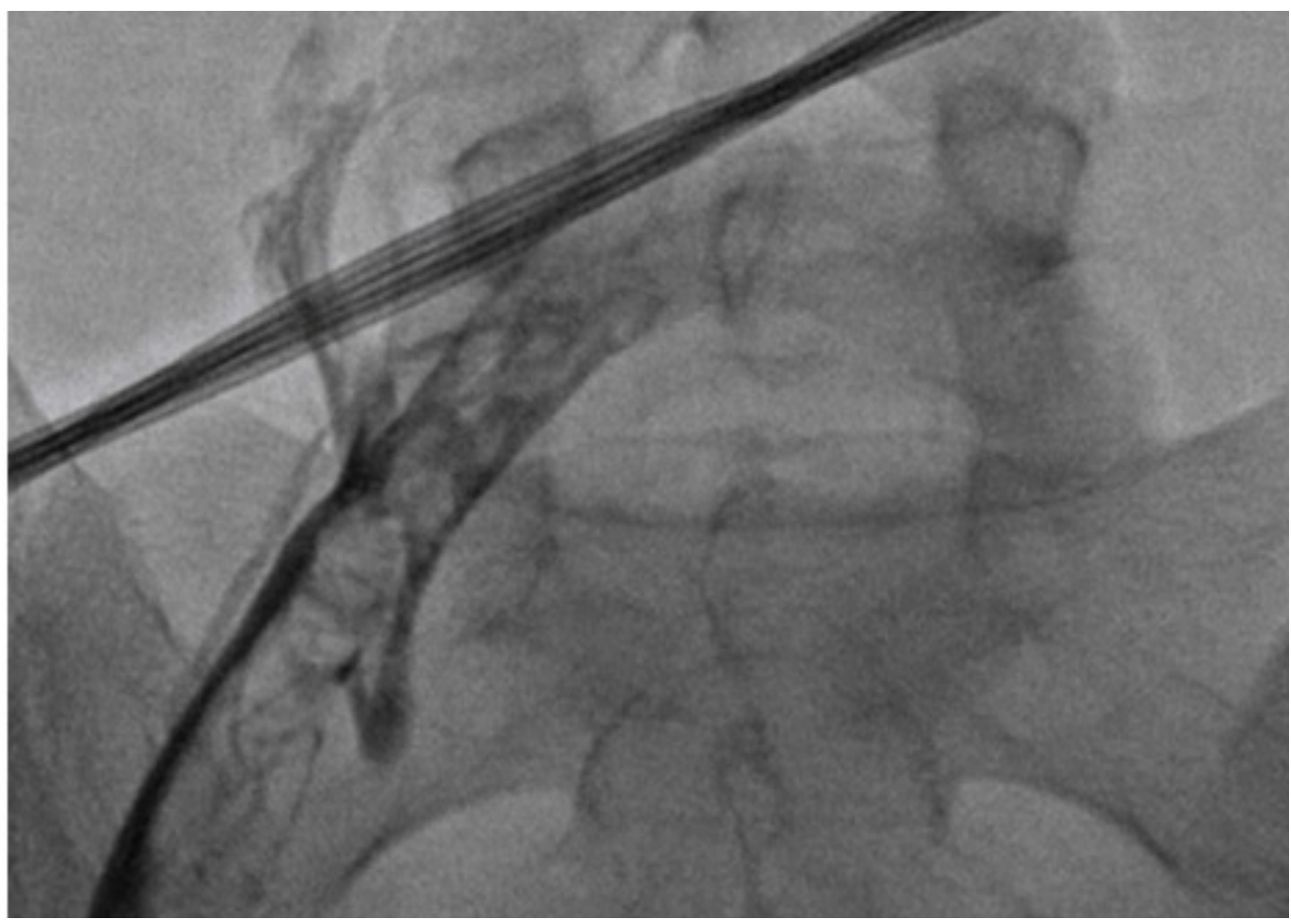

Figura 2. Imagen donde se observa trombo a nivel de transición de vena femoral e iliaca externa, con defecto de paso de medio de contraste durante venografía para trombolisis (imágenes autorizadas por servicio de hemodinamia, Fundación Valle del Lili). 
para definir posibilidad de trombectomía, que se encontró contraindicada por trombos en todo el eje iliaco izquierdo con drenaje por circulación colateral (figura 2), por lo cual se decidió continuar con anticoagulación.

El tiempo de hospitalización fue de 19 días; se dio de alta con rivaroxaban por orden de hematología debido a que se administra en una dosis diaria con menor tasa de resangrado y menor interacción medicamentosa (12). La paciente continuó en controles con clínica de anticoagulación, cirugía vascular y alta complejidad obstétrica durante dos meses después del parto, hubo resolución del cuadro.

\section{MATERIALES Y MÉTODOS}

Se realizó una revisión de la literatura registrada en las bases de datos Medline vía PubMed, Ovid, Embase, SciELO y Google Scholar desde el año 1997 hasta marzo del 2018, con los términos de búsqueda MeSH: "vena cava inferior”; "thrombosis"; "pregnancy complications" and "pregnancy trimester". Se incluyeron reportes de caso y series de casos en inglés y español. Se extrajo información sobre las siguientes variables: tipo de tratamiento (heparina no fraccionada, heparina de bajo peso molecular, trombolisis, filtro de vena cava inferior, tratamiento mixto); edad gestacional al momento del tratamiento; duración del tratamiento; información sobre el trabajo de parto; resultados del tratamiento antitrombótico (control de la TVP); resultados de riesgo de la terapia antitrombótica; complicaciones maternas y fetales; resultado materno y fetal definitivo; manejo posparto, y recurrencia de la ETV. Se excluyeron los estudios en los cuales no hubo acceso al texto completo o no mencionaban el tratamiento realizado.

Aspectos éticos. El presente trabajo fue avalado por el comité de ética médica de la Fundación Clínica Valle del Lili bajo el acta 20 del 5 de octubre del 2016. Se obtuvo el consentimiento informado de la paciente para la publicación de este reporte de caso y las imágenes que contiene. Se tomaron todas las precauciones para garantizar la confidencialidad de la información y su privacidad.

\section{RESULTADOS}

De un total de 185 títulos identificados, 17 estudios cumplieron con los criterios de búsqueda, de los cuales 2 fueron series de caso $(18,19)$ y 15 reportes de caso los cuales fueron revisados en texto completo (tabla 1). Se lograron identificar 41 casos constituidos por 35 pacientes con diagnóstico de trombosis de vena cava inferior durante el embarazo $(18,19,21-23,25,26,28,29,31,32)$ y 6 durante el puerperio $(20,24,27,30,33,34)$. En el $100 \%$ de las pacientes se decidió manejo anticoagulante con heparina de bajo peso molecular o heparina no fraccionada. En ocho reportes de caso decidieron anticoagular a las gestantes con heparina de bajo peso molecular antes del parto sin presentar complicaciones, tampoco se reportaron nuevos episodios de trombosis o sangrado (19, 20, 22, 23, 29, 30, 32).

Por otro lado, cuatro reportes de caso utilizaron heparina no fraccionada (HNF) seguida de inserción de filtro de vena cava inferior como tratamiento de elección (25-28). Lilijic et al. (27) reportaron un evento de trombosis profunda un año después de usar heparina no fraccionada en la paciente; sin embargo, no se reportaron otros tipos de complicaciones.

Una de las razones por las cuales optaron por HNF fue la reversión rápida de la anticoagulación debido a que la heparina se elimina de manera eficiente por su unión al endotelio. Opara, Jamjute, Lilijic y Milford et al. utilizaron HNF con una dosis de 5000 UI en bolo, seguido de una infusión de $15-25 \mathrm{ml} / \mathrm{kg}$; de igual forma, coincidieron en la interrupción de la anticoagulación antes del parto (25-28).Con respecto al uso de filtro de vena cava un total de 34 pacientes requirieron inserción de filtro de vena cava inferior $(18,19,21,23,25,26,29,30$ 33), una paciente requirió trombectomía (22), una gestante requirió trombolisis (34) y cinco mujeres recibieron antitrombóticos sin otra intervención asociada $(20,24,27,29,34)$.

Ocho autores reportaron la inserción del filtro de vena cava inferior antes del trabajo de parto en fase activa $(19,21,25,26,28,30,31)$; dos autores coincidieron en usar este elemento durante el 


\section{Tabla 1.}

Trombosis de vena cava inferior en el embarazo: búsqueda sistemática desde el año 1997 hasta el primer semestre del 2017

\begin{tabular}{|c|c|c|c|c|c|c|c|}
\hline$\stackrel{\stackrel{0}{3}}{\frac{b}{4}}$ & 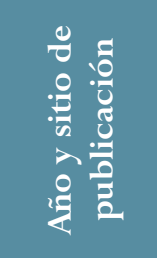 & 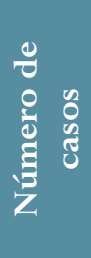 & 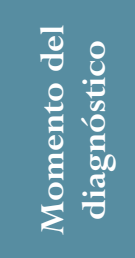 & 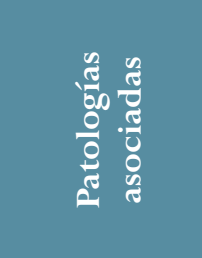 & 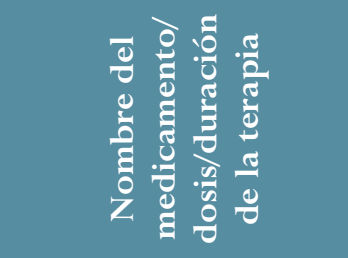 & 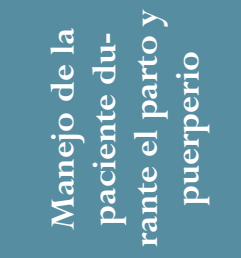 & 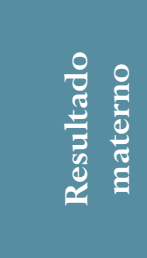 \\
\hline $\begin{array}{l}\text { Sherer } \\
\text { et al. (20) }\end{array}$ & $\begin{array}{l}1997, \\
\text { Estados } \\
\text { Unidos }\end{array}$ & 1 & Posparto & $\begin{array}{l}\text { Trombosis } \\
\text { de la vena } \\
\text { ovárica }\end{array}$ & $\begin{array}{l}\text { HBPM hasta el día } 23 \\
\text { posparto (sin dato de } \\
\text { dosis). A partir del día } \\
24 \text { posparto con war- } \\
\text { farina }\end{array}$ & $\begin{array}{l}\text { Parto sin } \\
\text { anticoagulación. } \\
\text { Puerperio con } \\
\text { uso de HBPM y } \\
\text { warfarina }\end{array}$ & $\begin{array}{l}\text { Sin } \\
\text { complica- } \\
\text { ciones }\end{array}$ \\
\hline $\begin{array}{l}\text { Rochelson } \\
\text { et al. ( } 21)\end{array}$ & $\begin{array}{l}2003, \\
\text { Estados } \\
\text { Unidos }\end{array}$ & 1 & $\begin{array}{l}18 \\
\text { semanas }\end{array}$ & $\begin{array}{l}\text { Ruptura } \\
\text { prematura de } \\
\text { membranas }\end{array}$ & $\begin{array}{l}\text { Anticoagulación e } \\
\text { inserción de filtro de } \\
\text { VCI antes del trabajo } \\
\text { de parto (sin dato de } \\
\text { medicamento y dosis de } \\
\text { la terapia) }\end{array}$ & $\begin{array}{l}\text { Antes del parto } \\
\text { se insertó el } \\
\text { filtro de VCI. } \\
\text { Sin dato de } \\
\text { terapia en el } \\
\text { puerperio }\end{array}$ & $\begin{array}{l}\text { Sin } \\
\text { complica- } \\
\text { ciones }\end{array}$ \\
\hline $\begin{array}{l}\text { Kawamata } \\
\text { Ket al. } \\
(18)\end{array}$ & $\begin{array}{l}2005, \\
\text { Osaka, } \\
\text { Japón }\end{array}$ & 11 & $\begin{array}{l}\text { Entre } \\
\text { las } 7-36 \\
\text { semanas }\end{array}$ & $\begin{array}{l}\text { Deficiencia } \\
\text { de proteína } \\
\text { S y síndrome } \\
\text { de anticoagu- } \\
\text { lante lúpico }\end{array}$ & $\begin{array}{l}\text { Dos semanas antes del } \\
\text { trabajo de parto trom- } \\
\text { bolisis con uroquinasa } \\
48.000 \mathrm{UI} / \text { día. Durante } \\
\text { el trabajo de parto en } \\
\text { tres pacientes se realiza } \\
\text { inserción de filtro de } \\
\text { VCI }\end{array}$ & $\begin{array}{l}\text { Durante el } \\
\text { trabajo de parto } \\
\text { en tres pacien- } \\
\text { tes se realiza } \\
\text { inserción de fil- } \\
\text { tro de VCI. Sin } \\
\text { datos de terapia } \\
\text { en el puerperio }\end{array}$ & $\begin{array}{l}\text { Sin } \\
\text { complica- } \\
\text { ciones }\end{array}$ \\
\hline $\begin{array}{l}\text { Gormus } \\
\text { et al. ( } 22)\end{array}$ & $\begin{array}{l}2005, \\
\text { Turquía }\end{array}$ & 1 & $\begin{array}{l}10 \\
\text { semanas }\end{array}$ & $\begin{array}{l}\text { Neuropatía } \\
\text { del nervio } \\
\text { ciático }\end{array}$ & $\begin{array}{l}\text { HBPM cada } 12 \text { horas } \\
\text { por dos días. Posterior- } \\
\text { mente trombectomía } \\
\text { por extensión de TVP } \\
\text { hasta vena renal }\end{array}$ & $\begin{array}{l}\text { HBPM cada } 12 \\
\text { horas durante el } \\
\text { mes posparto }\end{array}$ & $\begin{array}{l}\text { Sin } \\
\text { complica- } \\
\text { ciones }\end{array}$ \\
\hline $\begin{array}{l}\text { Cheung } \\
\text { et al. (23) }\end{array}$ & $\begin{array}{l}\text { 2005, } \\
\text { Canadá }\end{array}$ & 1 & $\begin{array}{l}35 \\
\text { semanas }\end{array}$ & Ninguna & $\begin{array}{l}\text { HBPM (dalteparina } 200 \\
\text { UI } / \mathrm{kg} \text { ) }\end{array}$ & $\begin{array}{l}\text { Filtro de VCI } \\
\text { durante el par- } \\
\text { to. Sin datos del } \\
\text { puerperio }\end{array}$ & $\begin{array}{l}\text { Sin } \\
\text { complica- } \\
\text { ciones }\end{array}$ \\
\hline $\begin{array}{l}\text { Pankaja } \\
\text { et al. (24) }\end{array}$ & $\begin{array}{l}2006, \\
\text { Inglaterra }\end{array}$ & 1 & Posparto & Ninguna & $\begin{array}{l}\text { Warfarina (sin datos } \\
\text { de dosis y duración del } \\
\text { tratamiento) }\end{array}$ & $\begin{array}{l}\text { Sin anticoagula- } \\
\text { ción durante el } \\
\text { parto. Inserción } \\
\text { de filtro de VCI } \\
\text { en el puerperio }\end{array}$ & $\begin{array}{l}\text { Sin } \\
\text { complica- } \\
\text { ciones }\end{array}$ \\
\hline $\begin{array}{l}\text { Opara } \\
\text { et al. }(25)\end{array}$ & $\begin{array}{l}2006, \\
\text { Inglaterra }\end{array}$ & 1 & $\begin{array}{l}37,5 \\
\text { semanas }\end{array}$ & $\begin{array}{l}\text { Soplo cardia- } \\
\text { co funcional } \\
\text { y síndrome } \\
\text { de intestino } \\
\text { irritable }\end{array}$ & $\begin{array}{l}\text { Heparina no fraccio- } \\
\text { nada bolo de } 5000, \text { UI } \\
\text { seguido de infusión de } \\
15-25 \mathrm{ml} / \mathrm{kg} \text { por hora } \\
\text { e inserción de filtro de } \\
\text { VCI }\end{array}$ & Sin dato & $\begin{array}{l}\text { Sin } \\
\text { complica- } \\
\text { ciones }\end{array}$ \\
\hline
\end{tabular}




\begin{tabular}{|c|c|c|c|c|c|c|c|}
\hline$\stackrel{\frac{o}{3}}{2}$ & 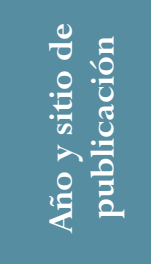 & 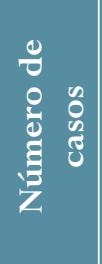 & 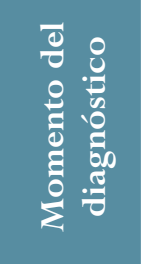 & 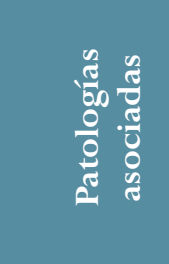 & 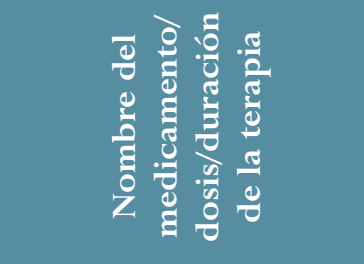 & 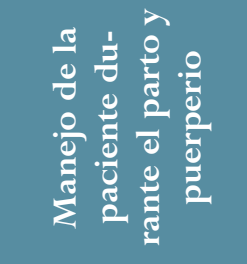 & 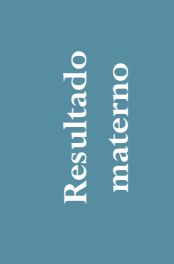 \\
\hline $\begin{array}{l}\text { Jamjute } \\
\text { et al. (26) }\end{array}$ & $\begin{array}{l}2006, \\
\text { Inglaterra }\end{array}$ & 1 & $\begin{array}{l}36 \\
\text { semanas }\end{array}$ & $\begin{array}{l}\text { No } \\
\text { mencionan }\end{array}$ & $\begin{array}{l}\text { Heparina no fraccionada } \\
\text { (sin dato de dosis y du- } \\
\text { ración) y posteriormente } \\
\text { antes del parto inserción } \\
\text { de filtro de VCI }\end{array}$ & Sin dato & $\begin{array}{l}\text { Sin } \\
\text { complica- } \\
\text { ciones }\end{array}$ \\
\hline $\begin{array}{l}\text { Ilijic et al. } \\
(27)\end{array}$ & $\begin{array}{l}2007, \\
\text { Croacia }\end{array}$ & 1 & Posparto & $\begin{array}{l}\text { Pielone- } \\
\text { fritis } \\
\text { bilateral }\end{array}$ & $\begin{array}{l}\text { Heparina no fraccionada } \\
\text { bolo de } 5000 \mathrm{UI} \text { seguido } \\
\text { de infusión } 1300 \mathrm{UI} / \mathrm{h} \\
\text { por } 5 \text { días, posterior- } \\
\text { mente HBPM } 60 \mathrm{mg}\end{array}$ & Sin dato & $\begin{array}{l}\text { Un año } \\
\text { después se } \\
\text { diagnosticó } \\
\text { nuevamente } \\
\text { TVP }\end{array}$ \\
\hline $\begin{array}{l}\text { Milford } \\
\text { et al. (28) }\end{array}$ & $\begin{array}{l}2009 \\
\text { Australia }\end{array}$ & 1 & $\begin{array}{l}39,4 \\
\text { semanas }\end{array}$ & Ninguna & $\begin{array}{l}\text { Heparina no fraccionada } \\
\text { bolo de } 5000 \text { UI seguido } \\
\text { de infusión e inserción } \\
\text { de filtro de VCI }\end{array}$ & $\begin{array}{l}12 \text { horas antes } \\
\text { del parto se } \\
\text { suspendió la he- } \\
\text { parina no frac- } \\
\text { cionada. Diez } \\
\text { horas después } \\
\text { del parto se ini- } \\
\text { ció enoxaparina } \\
90 \text { mg cada } 12 \\
\text { horas al día por } \\
\text { cinco días }\end{array}$ & $\begin{array}{l}\text { Sin } \\
\text { complica- } \\
\text { ciones }\end{array}$ \\
\hline $\begin{array}{l}\text { Fernández } \\
\text { et al. (29) }\end{array}$ & $\begin{array}{l}2010 \\
\text { México }\end{array}$ & 1 & $\begin{array}{l}41 \\
\text { semanas }\end{array}$ & Ninguna & $\begin{array}{l}\text { HBPM } 60 \text { mg antes del } \\
\text { parto. Durante el parto } \\
\text { heparina no fraccionada } \\
25000 \text { unidades }\end{array}$ & $\begin{array}{l}\text { Se suspendió } \\
\text { anticoagulación } \\
12 \text { horas antes } \\
\text { del parto. Dos } \\
\text { horas posparto } \\
\text { se inició hepa- } \\
\text { rina no fraccio- } \\
\text { nada por } 4 \text { días. } \\
\text { Fue dada de alta } \\
\text { con acenocu- } \\
\text { maron } 4 \text { mg y } \\
\text { HBPM } 40 \text { mg } \\
\text { cada } 24 \text { horas }\end{array}$ & $\begin{array}{l}\text { Sin } \\
\text { complica- } \\
\text { ciones }\end{array}$ \\
\hline $\begin{array}{l}\text { Wei et al. } \\
(30)\end{array}$ & $\begin{array}{l}2012, \\
\text { China }\end{array}$ & 1 & Posparto & Ninguna & $\begin{array}{l}\text { HBPM } 7500 \text { UI cada } \\
12 \text { horas e inserción de } \\
\text { filtro en VCI }\end{array}$ & $\begin{array}{l}\text { Uso de filtro de } \\
\text { VCI hasta el } 15 \\
\text { día posparto }\end{array}$ & $\begin{array}{l}\text { Sin } \\
\text { complica- } \\
\text { ciones }\end{array}$ \\
\hline $\begin{array}{l}\text { Liu et al. } \\
\text { (19) }\end{array}$ & $\begin{array}{l}\text { 2012, } \\
\text { China }\end{array}$ & 15 & $\begin{array}{l}\text { Entre } \\
\text { las } \\
34-40 \\
\text { semanas }\end{array}$ & Sin dato & $\begin{array}{l}\text { HBPM } 4100 \text { UI cada } \\
12 \text { horas e inserción de } \\
\text { filtro en VCI }\end{array}$ & $\begin{array}{l}\text { Se suspendió la } \\
\text { anticoagulación } \\
12 \text { horas antes } \\
\text { del parto y se } \\
\text { inició } 12 \text { horas } \\
\text { después }\end{array}$ & $\begin{array}{l}\text { Sin } \\
\text { complica- } \\
\text { ciones }\end{array}$ \\
\hline
\end{tabular}




\begin{tabular}{|c|c|c|c|c|c|c|c|}
\hline 龸 & 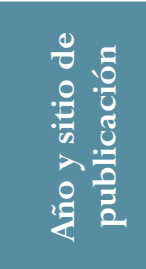 & $\begin{array}{l}0 \\
0 \\
0 \\
\text { है } \\
\text { है है } \\
\text { है } \\
\text { Z }\end{array}$ & 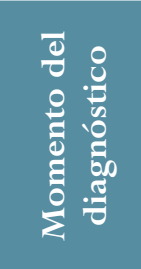 & 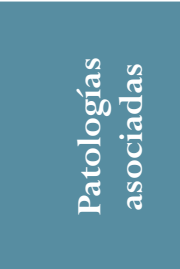 & 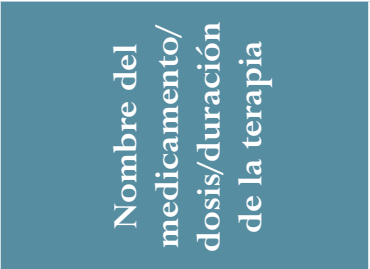 & 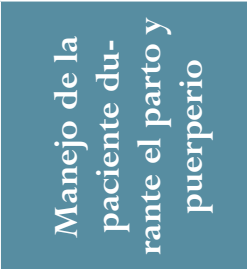 & 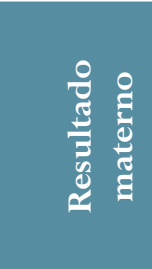 \\
\hline $\begin{array}{l}\text { Yamagami } \\
\text { et al. }(31)\end{array}$ & $\begin{array}{l}2013, \\
\text { Japón }\end{array}$ & 1 & $\begin{array}{l}34 \\
\text { semanas }\end{array}$ & Ninguna & $\begin{array}{l}\text { Filtro de VCI y warfarina } \\
\text { (sin dato de dosis) }\end{array}$ & $\begin{array}{l}\text { Tres días } \\
\text { después del } \\
\text { parto se inició } \\
\text { warfarina }\end{array}$ & $\begin{array}{l}\text { Sin } \\
\text { complica- } \\
\text { ciones }\end{array}$ \\
\hline $\begin{array}{l}\text { Plessis } \\
\text { et al. (32) }\end{array}$ & $\begin{array}{l}2016 \\
\text { Australia }\end{array}$ & 1 & $\begin{array}{l}6 \\
\text { semanas }\end{array}$ & Trombofilia & $\begin{array}{l}\text { HBPM } 80 \text { mg cada } 12 \\
\text { horas e inserción de } \\
\text { filtro en VCI }\end{array}$ & $\begin{array}{l}12 \text { horas antes } \\
\text { del parto se } \\
\text { suspendió anti- } \\
\text { coagulación y } 4 \\
\text { horas después } \\
\text { del parto se } \\
\text { inició HBPM }\end{array}$ & $\begin{array}{l}\text { Sin } \\
\text { complica- } \\
\text { ciones }\end{array}$ \\
\hline $\begin{array}{l}\text { Rimbas } \\
\text { RC et al. } \\
(33)\end{array}$ & $\begin{array}{l}2017 \\
\text { Romania }\end{array}$ & 1 & Posparto & Ninguna & $\begin{array}{l}\text { Trombolisis dirigida por } \\
\text { cateterismo (sin dato de } \\
\text { dosis y duración) }\end{array}$ & Sin dato & $\begin{array}{l}\text { Sin } \\
\text { complica- } \\
\text { ciones }\end{array}$ \\
\hline $\begin{array}{l}\text { Tashjian } \\
\text { et al. (34) }\end{array}$ & $\begin{array}{l}2017, \\
\text { Estados } \\
\text { Unidos }\end{array}$ & 1 & Posparto & $\begin{array}{l}\text { Cardiopatía } \\
\text { congénita }\end{array}$ & HBPM dos veces al día & $\begin{array}{l}4 \text { días después } \\
\text { del parto se sus- } \\
\text { pendió HBPM } \\
\text { y se inició } \\
\text { warfarina }\end{array}$ & $\begin{array}{l}\text { Sin } \\
\text { complica- } \\
\text { ciones }\end{array}$ \\
\hline
\end{tabular}

VCI: vena cava inferior; HPBM: heparina de bajo peso molecular

trabajo de parto $(18,23)$, y solo Pankaja et al. lo utilizaron durante el puerperio (24). La colocación de este elemento se realizó con el fin de lograr suspender de manera segura la anticoagulación durante el parto y así evitar complicaciones hemorrágicas, pero teniendo en cuenta la aparición de embolia pulmonar que puede ser provocada al retirar la anticoagulación. Cheung et al., en su serie de casos, documentaron que la inserción de filtro de vena cava podría ser efectiva en el tratamiento anticoagulante (23).

En cuanto al uso de agentes trombolíticos, Kawamata et al. (18) y Rimbas et al. (33) utilizaron la trombolisis como tratamiento en sus pacientes, ambos reportes utilizaron la uroquinasa debido a que mimetiza las moléculas antitrombóticas naturales.

\section{CONCLUSIONES}

Existen varias opciones terapéuticas en el tratamiento de la trombosis profunda de la vena cava durante el embarazo: heparina de bajo peso molecular, heparina no fraccionada, trombolisis y filtro de vena cava inferior; cada una de estas opciones tiene ventajas y riesgos. La elección del tratamiento más adecuado depende de cada paciente, se deben tener en cuenta los factores de riesgo y los antecedentes de las gestantes. El manejo adecuado de esta patología durante el embarazo es un reto para los equipos médicos en los niveles de alta complejidad y requiere más evidencia científica que soporte una posición clara.

\section{REFERENCIAS}

1. Marik PE, Plante LA. Venous thromboembolic disease and pregnancy. N Engl J Med. 2008;359:2025-33. https://doi.org/10.1056/NEJMra0707993

2. Bourjeily G, Paidas M, Khalil H, Rosene-Montella K, Rodger M. Pulmonary embolism in pregnancy. The Lancet. 2010;375:500-12. https://doi.org/10.1016/ S0140-6736(09)60996-X 
3. Middeldorp S. Thrombosis in women: What are the knowledge gaps in 2013? J Thromb Haemost (JTH). 2013;11 Suppl 1:180-91. https://doi.org/10.1111/ jth. 12266

4. Stein PD, Beemath A, Matta F, Weg JG, Yusen RD, Hales CA, et al. Clinical characteristics of patients with acute pulmonary embolism: Data from PIOPED II. Am J Med. 2007;120:871-9. https://doi. org/10.1016/j.amjmed.2007.03.024

5. Heit JA, et al. Trends in the incidence of venous thromboembolism during pregnancy or postpartum: A 30-year population-based study. Ann Intern Med. 2005;143:697-706. https://doi.org/10.7326/00034819-143-10-200511150-00006

6. Pomp ER, Lenselink AM, Rosendaal FR, Doggen CJM. Pregnancy, the postpartum period and prothrombotic defects: Risk of venous thrombosis in the MEGA study. J Thromb Haemost JTH. 2008;6:632-7. https://doi. org/10.1111/j.1538-7836.2008.02921.x

7. Jacobsen AF, Skjeldestad FE, Sandset PM. Incidence and risk patterns of venous thromboembolism in pregnancy and puerperium — a register-based casecontrol study. Am J Obstet Gynecol. 2008;198:233. e1-7. https://doi.org/10.1016/j.ajog.2007.08.041

8. Rosenberg VA, Lockwood CJ. Thromboembolism in pregnancy. Obstet Gynecol Clin North Am. 2007;34:481-500, xi. https://doi.org/10.1016/j. ogc.2007.06.006

9. Nelson SM, Greer IA. Thrombophilia and the risk for venous thromboembolism during pregnancy, delivery, and puerperium. Obstet Gynecol Clin North Am. 2006;33:413-27. https://doi.org/10.1016/j. ogc.2006.05.009

10. Doyle NM, Monga M. Thromboembolic disease in pregnancy. Obstet Gynecol Clin North Am. 2004;31:319-44, vi. https://doi.org/10.1016/j. ogc. 2004.03.008

11. Gherman RB, Goodwin TM, Leung B, Byrne JD, Hethumumi R, Montoro M. Incidence, clinical characteristics, and timing of objectively diagnosed venous thromboembolism during pregnancy. Obstet Gynecol. 1999;94(5 Pt 1):730-4. https://doi. org/10.1097/00006250-199911000-00017

12. Ginsberg JS, Hirtsh J. Use of antithrombotic agents during pregnancy. Chest 1995;108:suppl: 305S-11S. https://doi.org/10.1378/chest.108.4_ Supplement.305S
13. Ferrer MF, Oyarzun EE. Trombosis venosa en el embarazo. Revista médica Clínica las Condes. 2014;25:1004-18. https://doi.org/10.1016/S07168640(14)70650-9

14. Hirsh J. Heparin. N Engl J Med. 1991;324:1565-74. https://doi.org/10.1056/NEJM199105303242206

15 Ginsberg JS, Bates SM. Management of venous thromboembolism during pregnancy. J Thromb Haemost. 2003;1:1435-42. https://doi.org/10.1046/ j.1538-7836.2003.00307.x

16. Gartman EJ. The use of thrombolytic therapy in pregnancy. Obstet Med. 2013; 6:105-11. https://doi. org/10.1177/1753495X13488771

17. Oami T, Oshima T, Oku R, Nakanishi K. Successful treatment of pulmonary embolism-induced cardiac arrest by thrombolysis and targeted temperature management during pregnancy. Acute Med Surg. 2018;5:292-5. https://doi.org/10.1002/ams2.345

18. Kawamata K, Chiba Y, Tanaka R, Higashi M, Nishigami $\mathrm{K}$. Experience of temporary inferior vena cava filters inserted in the perinatal period to prevent pulmonary embolism in pregnant women with deep vein thrombosis. J Vasc Surg. 2005;41:652-6. https://doi. org/10.1016/j.jvs.2005.01.023

19. Liu Y, Sun Y, Zhang S, Jin X. Placement of a retrievable inferior vena cava filter for deep venous thrombosis in term pregnancy. J Vasc Surg. 2012;55:1042-7. https:// doi.org/10.1016/j.jvs.2011.10.107

20. Sherer DM, Fern S, Mester J, Barnhard Y, Divon MY. Postpartum ultrasonographic diagnosis of inferior vena cava thrombus associated with ovarian vein thrombosis. Am J Obstet Gynecol. 1997;177:474-5. https://doi.org/10.1016/S0002-9378(97)70224-2

21. Rochelson B, Scher L, Warshawsky R, Simon D. Use of a temporary vena cava filter in a woman with septic abortion and inferior vena cava thrombosis. A case report. J Reprod Med. 2003;48:557-9.

22. Gormus N, Ustun ME, Paksoy Y, Ogun TC, Solak H. Acute thrombosis of inferior vena cava in a pregnant woman presenting with sciatica: A case report. Ann Vasc Surg. 2005;19:120-2. https://doi.org/10.1007/ s10016-004-0142-2.

23. Cheung MC, Asch MR, Gandhi S, Kingdom JCP. Temporary inferior vena caval filter use in pregnancy. J Thromb Haemost JTH. 2005;3:1096-7. https://doi. org/10.1111/j.1538-7836.2005.01299.x 
24. Pankaja S, Osborne K, Pringle S. Extensive bilateral iliofemoral and inferior vena cava thrombosis following caesarean section. J Obstet Gynaecol. 2006;26:820-1. https://doi.org/10.1080/01443610600987191

25. Opara E, Gile J, Zaidi J. Inferior vena cava filter thrombo-prophylaxis in high-risk twin pregnancy. Int J Gynaecol Obstet. 2007;98:62-3. https://doi. org/10.1016/j.ijgo.2006.11.027

26. Jamjute P, Reed N, Hinwood D. Use of inferior vena cava filters in thromboembolic disease during labor: Case report with a literature review. J MaternFetal Neonatal Med. 2006;19:741-4. https://doi. org/10.1080/14767050600631443

27. Ilijic M, Ivanisevic M, Djelmis J, Krpan M, Banfic L, Lusic M. Postpartal deep-vein thrombosis revealing agenesis of the inferior vena cava. Eur J Obstet Gynecol Reprod Biol. 2007;131:235-6. https://doi. org/10.1016/j.ejogrb.2006.04.003.

28. Milford W, Chadha Y, Lust K. Use of a retrievable inferior vena cava filter in term pregnancy: Case report and review of literature. Aust N Z J Obstet Gynaecol. 2009;49:331-3. https://doi.org/10.1111/j.1479828X.2009.01004.x

29. Fernández E, Due-as N, Villafranca A, Perea M, Amorós B, Bermejo L. Gestante a término con trombosis de la vena cava inferior: tratamiento anestésico en el parto. Rev Esp Anestesiol Reanim. 2010;57:307-10. https://doi.org/10.1016/S0034-9356(10)70232-2
30. Wei Y, Ouyang P, Yang W. High inferior vena cava thrombosis in a 16-year-old postpartum patient: A case report. J Huazhong Univ Sci Technol Med Sci. 2012;32:149-50. https://doi.org/10.1007/s11596012-0027-5

31. Yamagami T, Yoshimatsu R, Okubo T, Tanaka O, Miura H, Yamada K, et al. Symptomatic deep venous thrombosis of the lower extremity in a pregnant woman successfully treated with endovascular procedures. J Obstet Gynaecol Res. 2013;39:1059-64. https://doi.org/10.1111/j.1447-0756.2012.02071.x

32. Du Plessis LE, Mol BW, Svigos JM. The use of retrievable inferior vena cava filters in pregnancy: Another successful case report, but are we actually making a difference? Obstet Med. 2016;9:102-5. https://doi.org/10.1177/1753495X16648026

33. Rimbas RC, Câlin SI, Ionescu H, Dorobat B, Vinereanu $D$, Cinteza M. An extensive suprarenal inferior vena cava thrombosis successfully treated with catheterdirected thrombolysis in a postpartum 14-year-old girl. VASA Z Gefasskrankheiten. 2017;46:227-30. https://doi.org/10.1024/0301-1526/a000605

34. Tashjian JA, Fraint H, DiNardo J, Rouine-Rapp K. Inferior vena cava thrombus in a postpartum patient with fontan physiology: A case report. A A Case Rep. 2017;9:136-9. 